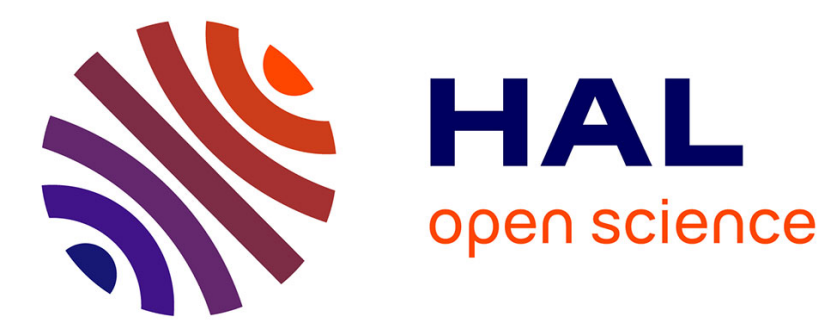

\title{
Mode digitale en tension, de l'intime au public
}

Lucile Salesses, Marie Ouvrard-Servanton

\section{To cite this version:}

Lucile Salesses, Marie Ouvrard-Servanton. Mode digitale en tension, de l'intime au public. Sociétés Revue des sciences sociales et humaines, 2017. hal-01775091

\section{HAL Id: hal-01775091 https://hal-amu.archives-ouvertes.fr/hal-01775091}

Submitted on 24 Apr 2018

HAL is a multi-disciplinary open access archive for the deposit and dissemination of scientific research documents, whether they are published or not. The documents may come from teaching and research institutions in France or abroad, or from public or private research centers.
L'archive ouverte pluridisciplinaire HAL, est destinée au dépôt et à la diffusion de documents scientifiques de niveau recherche, publiés ou non, émanant des établissements d'enseignement et de recherche français ou étrangers, des laboratoires publics ou privés. 


\title{
Mode digitale en tension, de l'intime au public
}

\author{
Lucile SALESSES, Maître de conférences \\ Aix-Marseille Univ, LPS, Aix-en-Provence, France \\ Marie OUVRARD-SERVANTON, Maître de conférences \\ Aix-Marseille Univ, ADEF, Marseille, France
}

Résumé : Les liens entre la mode et la culture digitale questionnent les nouveaux canaux de distribution et de médiatisation des marques et tendances à l'ère du numérique ainsi que les expériences consommatoires découlant de ce processus de digitalisation de la mode. Dans ce contexte émergent, de grandes mutations opèrent un renouvellement des sources de création et des vecteurs d'influence : réseaux sociaux, blogs, sites de marques, recomposent le paysage traditionnel de la mode et ses imaginaires, pour le transformer en un écosystème digital dans lequel le consommateur devient partie prenante, entre l'intime et le public.

Mots clés : mode digitale, réseaux sociaux, blog, marque, image, imaginaire

\begin{abstract}
The links between fashion and digital culture are questioning the new distribution channels and medialization of the brands and the trends to the era of the digital technology. It is also questioning the customer's experiences ensuing from this process of digitalization of fashion. In this emergent context, great shifts operate a renewal of the sources of creation and the vectors of influence: social networks, blogs, brand websites. Traditional landscape of fashion and its imaginary representations are recomposed to be transformed into a digital ecosystem in which the consumer becomes stakeholder, between intimate and public area.
\end{abstract}

Keywords: digital fashion, social networks, blog, brand, image, imaginary

Avec la démocratisation d'Internet, le déploiement des médias sociaux et des terminaux mobiles, se développe l'espace digital et avec lui, s'installe un changement de paradigme inscrit dans la reconfiguration technologique, économique, sociale, et cognitive des sociétés. L'usage des surfaces digitales produit des modifications de fond dans les rituels de consommation, bouleverse les modèles de distribution et les politiques marketing. Internet est aujourd'hui un canal incontournable pour l'aide à la décision d'achat. Parmi les internautes français, $88 \%$ déclarent consulter des avis de consommateurs, des forums ou des blogs avant de réaliser un achat en ligne et $73 \%$ avant un achat en boutique. Par ailleurs, $52 \%$ dont $70 \%$

'Enseignant-chercheur Aix Marseille Univ, EA4671 ADEF, Aix-en-Provence, France. 
des 18/24 ans et $66 \%$ des 25-34 ans, consultent des avis sur les réseaux sociaux avant d'acheter en ligne. L'e-réputation devient un reflexe naturel d'information dont l'influence est multicanale dans la mesure où près de $90 \%$ des répondants se renseignent sur Internet lorsqu'ils sont intéressés par une publicité à la télévision ou un prospectus, signe que l'environnement digital a créé un autre consommateur'. Un consommateur faiseur de paroles et d'imaginaires sur le quotidien, l'ordinaire, l'exposition de soi. Autrement dit, un consommateur familier, s'exposant, se livrant, élaborant une identité numérique qu'il choisit de révéler, basculant ainsi de l'intime vers le public, s'inscrivant par là même dans une nouvelle norme d'apprentissage social, dont la visée est bien souvent la reconnaissance sociale ${ }^{3}$. Face à ce consommateur connecté, se déploie toute une sphère commerciale qui le scrute, l'utilise, l'influence, tente de le manipuler. Goûts, opinions, pratiques et imaginaires sont auscultés, traduits, recomposés, utilisés. Dans cet écosystème numérique, le système de la mode impénétrable il y a peu, inscrit désormais dans les pratiques digitales, se révèle, se rend visible y compris dans ses coulisses. Du secret, prisé dans ce secteur, à la diffusion publique des informations, il est en tension dans le même processus que son public. Comment se positionner? Comme faire lien ? Jusqu'où se dévoiler ? À partir de l'analyse du processus de digitalisation de la mode et des imaginaires associés, nous cherchons à répondre à ces questions, à montrer l'interrelation des acteurs entre puissance et vulnérabilité.

\section{Le numérique, amplificateur d'imaginaire}

Le prolongement dans l'espace numérique des relations entre marques et publics s'apparente à un construit social numérique découlant des réactions cumulatives des consommateurs aux informations diffusées dans l'espace digital. L'ensemble des réactions traduit les perceptions, images, attitudes et représentations que se font de la marque les consommateurs avertis ou non. La e-réputation s'inscrit alors dans une dynamique d'influence réciproque avec les consommateurs et nécessite pour les marques concernées de se situer dans la veille et l'analyse de l'image numérique. Cela suppose également d'intégrer l'idée que le consommateur est susceptible d'intervenir à toutes les étapes de la création d'un produit. Le digital conduit par conséquent les marques à être plus à l'écoute, à solliciter l'avis du consommateur sur les produits, leur fabrication et leur mise en scène, à tenir compte de ses attentes, à répondre à ses questions sur les responsabilités sociales et éthiques.

Le pouvoir de prescription de l'activité numérique se développe donc fortement. À l'apogée des réseaux sociaux, les consommateurs connectés sont activistes, plus que jamais ils sont consom'acteurs. ${ }^{4}$ Ils s'informent, discutent, mettent en cause, testent, participent, informent, forment, financent, partagent, persuadent. Internet est un amplificateur. Les choix effectués

\footnotetext{
http://www.reputationvip.com/ consulté le 05 février 2017 http://www.reputationvip.com/fr/blog/sondage-ifopreputation-vip-linfluence-de-le-reputation-sur-lacte-dachat ; http://www.ifop.com consulté le 05 février $2017 \mathrm{http} / / / \mathrm{www}$.ifop.com/?option=com publication\&type=poll\&id=2968

M. Ferraris, Mobilisation totale, PUF, Paris, 2016

‘ Voir E. Rémy, 2007, pour une définition du consom'acteur.
} 
par une marque peuvent être relayés, partagés, commentés par des milliers d'internautes. Les réseaux numériques, en permettant une divulgation de contenu immédiate, localisée et interactive, sont un lieu privilégié de création, de co-création, de diffusion et d'évolution des représentations et des imaginaires, ces productions mentales ou concrétisées par les images visuelles ou langagières produisant du sens (Wunenburger, 2003). L'imaginaire digital relève par conséquent de relations complexes entre savoirs investis d'affects (pathos), d'arguments rationnels (logos) ou d'images de soi (ethos) (Charaudeau, 2007), de représentations, de symboles, d'images, de valeurs, d'idéaux, d'utopies.

La notion d'imaginaire est définie dans la littérature scientifique à partir de deux grandes visions dont l'une est réduite et stable et l'autre étendue et dynamique (Wunenburger, 2003). La première conception de l'imaginaire l'appréhende comme le produit de l'imagination alors que pour la deuxième, il est dynamique, créatif et organisateur des images (Thomas, 1998) qui prennent sens dans la relation interactionnelle (Grassi, 2005). C'est cette orientation qui nous permet de dire que les images de mode au sens large déployées dans la sphère digitale, en s'inscrivant dans un imaginaire de la mode créatif et anticipateur structuré en un ensemble de représentations, génèrent à leur tour des représentations sociales structurantes de l'imaginaire social, participant ainsi à l'évolution de certaines d'entre elles ou encore à la création ou mutation de nouvelles valeurs.

Dans l'industrie de la mode, indiscutablement, la plus visuelle, expérientielle et esthétique de toutes, l'imaginaire occupe une place prépondérante, développant à partir de l'expérience humaine, des rites, des croyances et des perceptions, donc des significations communes et des capacités de création d'un collectif ${ }^{5}$. L'ensemble des images de mode fournissent à l'inconscient, couleurs, formes, symboles et modèles sociaux, qui déterminent les manières vestimentaires d'être et de paraître'. La transition anthropologique du digital s'exprime donc tout particulièrement dans ce secteur d'activité où l'essentiel se donne à voir dans les images.

\section{La digitalisation de la mode}

Les marques de mode s'adaptent à ce monde virtuel. La mode digitale provient certes d'une évolution technologique mais elle émane également de la compréhension des besoins de la société actuelle. Les hommes ont fait entrer le numérique dans leur vie, et les marques ont inclus cette stratégie à leur plan marketing.

L'économie de la numérisation provoque un changement culturel profond et le

${ }^{s}$ L. Salesses, D. Romain, The imaginary and social representations generated by fashion images in women's magazines. Papers on Social Representations, Volume 23, pages 23.1-23.20, 2014.

'E. Coutant, Pour une courte sociologie de la mode, dans Management et Marketing de la Mode, (dir.) Salesses,

L. ,Paris, Dunod, 2013. 
bouleversement de nombreux business-models. Les marques de mode se dotent a minima d'un site Web, d'un compte Facebook correctement géré, d'un fil Twitter, d'un lien Instagram alimenté, d'un site de vente en ligne'. Elles auscultent les communications des influenceurs du Web qu'ils soient alerteurs, tendanceurs, experts, évaluateurs. Autant de précurseurs et d'amplificateurs de tendances que sont les youtubeurs, bloggeurs, instagrameurs, vineurs, etc. Dans l'industrie de la mode, la stratégie multicanal est quasiment généralisée ${ }^{8}$, avec un pourcentage d'entreprises disposant de leur propre site Web'supérieur à $80 \%$. Dans ce secteur d'activité, les consommateurs ont particulièrement besoin d'échanger, de commenter, de faire part de leurs avis, de raconter, de se raconter, de se mettre en scène et de scénariser leurs achats. La relation à la marque, de nature socio-sémiotique, s'inscrit alors dans une expérience créative et socialisée online.

En effet, la création ne s'appuie plus uniquement sur les stylistes et modélistes, le partage des informations numériques implique la participation des responsables de collections, directeurs artistiques, sourceurs, chefs de produits, équipes marketing, data scientists, responsables de production, fournisseurs, sous-traitants et consommateurs. Ceux-ci par le biais des réseaux sociaux sont amenés à donner leur avis à toutes les étapes de la vie d'un produit et les entreprises mettent en place des stratégies de communication visant à susciter leur créativité, les impliquant en temps réel dans le processus créatif et d'évolution. En ce sens, la sphère digitale, permet l'ouverture des frontières et l'instantanéité des échanges, le partage d'informations, la transparence et, oblige à l'écoute.

L'ensemble des étapes de fonctionnement de la marque, autrefois non exposées, voire stratégiquement tenues secrètes, sont, du fait du digital, visibles, scénarisées, elles ont migré de l'intime au public. L'écosystème digital conduit les marques à re-présenter leur identité, à développer à leur propos du storytelling, à s'astreindre au plus de transparence possible, à s'employer à convaincre de leur attitude éthique et morale, à se dévoiler dans leur intimité, y compris celle des acteurs internes ${ }^{10}$. Cette conjointe évolution de la culture créative et de la culture numérique, conduit les marques à une relation de très grande proximité avec le consommateur, sollicitant son avis aussi bien sur la création d'un produit, sa fabrication, sa personnalisation, son recyclage, la façon dont il sera vendu, photographié, mis en scène. Ce rapprochement avec le consommateur crée de la valeur ajoutée pour les marques et il permet, dans le même temps, de contrôler et de réagir dans un espace temps minimalisé aux

L. Salesses, M. Ouvrard-Servanton, M., C. Alcantara, Marques de mode, influenceurs et e-réputation : vers une modélisation des relations entre capital marque, représentations et influence. 5ème édition du Webinaire «Eréputation et influenceurs: de nouveaux enjeux pour les organisations? », colloque scientifique organisé par l'Observatoire des médias sociaux en relations publiques de l'université Laval, co-organisé avec l'IDETCOM de l'Université de Toulouse 1 Capitole, MICA de l'Université de Bordeaux Montaigne et Elico de SciencesPoLyon. Québec, Canada, 5 avril 2016.

http://www.lesechos.fr/17/03/2014/lesechos.fr/0203378985896 les-grandes-marques-de-mode-a-l-assaut-de-latoile.htm\#3xR1DPGgU5Dxzub3.99 - Consulté le 10 février 2017.

"Selon une enquête réalisée par modaes.es, leader espagnol de l'information économique, spécialisé dans le secteur de la mode.

${ }^{10} \mathrm{Cf}$. la présence d'Olivier Rousteing sur les réseaux sociaux, et l'utilisation de ce levier par la griffe Balmain. 
éventuelles réactions négatives.

\section{De l'importance des algorithmes}

La fréquentation digitale laisse en toute conscience ou non de la part des internautes, des traces, des données exploitables par les data scientists et les marketeurs, dont l'objectif est de renforcer les liens entretenus avec le consommateur, d'approfondir les connaissances le concernant, de mieux connaître l'environnement économique et ses opportunités afin de déployer des solutions opérationnelles appropriées.

Dans un futur proche, la plupart des enseignes géo-localiseront leurs clients de manière à personnaliser l'offre à la situation géographique et au climat. Elles seront à même de lier le niveau de satisfaction du consommateur à son comportement d'achat, en mesure d'analyser les ventes en fonction de la météo. De même, elles seront capables de déterminer les produits à proposer à un client dont les habitudes d'achat sont restreintes au magasin, afin de l'inciter à acheter en ligne.

$\mathrm{Ce}$, au risque d'être perçues comme intrusives", ce qui est le cas pour 60,5\% des Français en 2016, alors qu'ils n'étaient que $29 \%$ à le déclarer en 2008. Cette sensibilité au risque de contrôle déjà relevée par Proulx et Kwok Choon en 2011'2 se confirme aujourd'hui. En effet, $72 \%$ se déclarent dérangés par le fait que des informations relatives à leur vie privée soient enregistrées dans une base de données et $66 \%$ ne tolèrent pas que les marques puissent accéder à des données issues des réseaux sociaux. Autrement dit, un quotidien perçu comme dominé par une logique marchande, conduit à redire l'importance du corps individuel dans le cadre du corps collectif.

Or, l'industrie de la mode avec des entreprises certes fortement mobilisées dans le ecommerce depuis plusieurs années mais plus frileuses quant à la numérisation des outils, se réorganise progressivement et en profondeur afin de s'insérer dans l'économie numérique. Les entreprises du secteur, confrontées à des enjeux forts en termes d'image et de désirabilité, s'inscrivent dans un changement d'ère, explorant les nouvelles opportunités dans le domaine du numérique, innovant en projets digitaux. Donner un nouveau tournant aux marques en matière de communication, nécessite pour elles de se consacrer autant aux réseaux sociaux qu'au domaine de la création et du style.

\footnotetext{
" $6^{\mathrm{mm}}$ édition du baromètre de l'intrusion, Publicis ETO et Toluna, janvier 2016

${ }^{12}$ S. Proulx, S. et M. J. Kwok Choon, «L'usage des réseaux socionumériques : une intériorisation douce et progressive du contrôle social », Hermès, $n^{\circ}$ 59, 2011, p. 105-111.

${ }^{13} \mathrm{Au}$ sens de M. Maffesoli, Le réenchantement du monde, Editions de la Table Ronde, 2007.
} 


\section{La mode et les réseaux sociaux}

Fin 2016, sur 3,773 milliards d'internautes, 2,789 milliards de personnes sont présentes sur les réseaux sociaux et sur ces 2,789 milliards, 2,549 milliards sont actives sur leur mobile ${ }^{14}$. Les réseaux sociaux se sont donc imposés comme un canal de communication incontournable pour les entreprises et ont donné lieu à la mise en place de stratégies marketing adaptées. Les consommateurs quant à eux, avec la pratique des réseaux sociaux, sont de plus en plus connectés. Ce canal de communication «naturel» pour eux, constitue le moyen de contacter rapidement leurs marques préférées en postant des commentaires, des likes, attendant en retour une réaction rapide de celles-ci. Ils ont donc des exigences qualitatives et temporelles, souhaitant obtenir dans l'instantané, des réponses à leurs commentaires et interrogations. Les entreprises doivent par conséquent se montrer présentes, réactives et veiller à leur image sur les réseaux sociaux, lesquels deviennent un élément clé de la relation clients digitale.

Ce faisant, le développement des réseaux sociaux numériques constitue une réelle opportunité pour les marques. Ils transforment les structures des relations sociales telles que définies par Simmel (1917) $)^{\text {s }}$, les formes d'organisation, les modes de conception et de commercialisation des biens et services, ils modifient le positionnement et les pratiques du consommateur, introduisant l'immédiateté, la proximité et le contact permanent avec celui-ci. Avec le développement des réseaux sociaux, l'installation des téléphones mobiles et la diffusion des outils permettant la création et la gestion de blogs, de nouveaux intermédiaires sont apparus, favorisant la mutation du système de la mode ${ }^{16}$, par l'interpénétration progressive entre les systèmes médiatiques traditionnels et les pratiques émergentes. Les blogs de mode, autonomes à leurs débuts, ont contribué à casser les codes relationnels entre les médias traditionnels, les marques et leurs publics. De contributions issues d'une passion pour la mode, inspirées du quotidien, de l'ordinaire, du partage d'expériences, inscrites dans la proximité, a émergé un nouveau format médiatique, un nouvel imaginaire, une nouvelle source d'influence.

\section{Le blog, un outil d'inter- fluence}

Le blog, devenu un média à part entière, est une plateforme qui permet à tout internaute de partager du contenu : texte, audiovisuel, photos et vidéos, d'exprimer des opinions, de livrer des expériences, de proposer un imaginaire. La blogosphère constitue un phénomène international à fort impact social, culturel, médiatique et économique. Les blogueurs, professionnels ou non, deviennent pour certains de véritables prescripteurs, que les marques, annonceurs et agences de communication considèrent comme des éléments clés de leurs

\footnotetext{
"http://www.blogdumoderateur.com/etat-lieux-2017-internet-reseaux-sociaux/. Page consultée le 6/2/2017.

" $\mathrm{M}$. Forse, Les réseaux sociaux chez Simmel : les fondements d'un modèle individualiste et structural, in L.

Deroche Gurcel et P. Watier (dir.), La Sociologie de Georges Simmel, Paris, PUF, 2002

${ }^{16}$ Pour reprendre l'expression de R. Barthes, 1967.
} 
stratégies.

Dans le domaine de la mode, c'est dans le courant des années 2000 que les premières blogueuses de mode s'imposent, en faisant un métier à part entière, comme Garance Doré, La Revue de Kenza ou encore le Blog de Betty. C'est au même moment que la blogosphère masculine émerge également, avec des blogs comme BonneGueule ou encore Comme un Camion. Ces blogs de mode mêlent, écrits, images (fixes et mouvantes) et sons, mettent en avant l'ordinaire des pratiques et des acteurs de la mode, privilégiant un ton spontané, un contenu subjectif et l'irrévérence'"

Les blogs sont aujourd'hui monétisés à l'instar des médias traditionnels. Les marques utilisent ces nouveaux influenceurs pour leur publicité, ayant recours à des leaders d'opinions, prisés des jeunes et reconnus sur la toile à travers le monde. Les médias blogs constituent un marché à part entière et certains, à forte audience, font appel à des régies comme Talent Agency ou encore Glam Media pour la commercialisation des espaces publicitaires. Les méthodes de monétisation des blogs, tout en reprenant celles de sites traditionnels comme la diffusion de bannières publicitaires et de liens d'affiliation, ajoutent la rédaction et diffusion de «billets » sponsorisés par les marques partenaires. Ces articles rédigés à la demande d'un annonceur, sur la marque, le produit ou le service, sont en lien avec la thématique du blog et si toutefois le blogueur est libre dans la rédaction du contenu et le choix des images, l'annonceur, en fonction de l'accord qui a été préalablement conclu, peut avoir un droit de regard.

Ainsi, le blog, outil d'expression de soi, d'expression du sujet parlant, ${ }^{18}$ d'autopublication, producteur de contenus, d'une identité personnelle et intime, dévoile l'auteur tout en l'inscrivant de manière synchrone dans l'interactivité, le faisant tisseur de liens, créateur d'imaginaires et outil de communication collective. L'accès à l'information immédiate, alternative, mais également contributive, propositionnelle, génère sentiment d'appartenance à une communauté, considération, relations de confiance. L'information se transforme en conversation intime, perçue comme telle, alors que publique. Aussi, inévitablement, la monétisation des blogs de mode a fait polémique, entrainant déception et critiques. Les frontières entre la sphère économique et la sphère "privée» devenaient poreuses, constituaient une zone grise et déformée ${ }^{19}$, perdant de sa netteté. Non plus subjective, flottante, spontanée, l'expression produite par l'un, devenait suspecte avant que d'être commentée par l'autre. Les codes du système relationnel établi étant bouleversés, le processus de complémentarité développé par Maffesoli $(2007,2016)$ est en partie nié et non reconnu. Comment en conséquence être soi-même en fonction de l'altérité si celle-ci est masquée pour

\footnotetext{
"A. Rocamora, D. Bartlett, «Blogs de mode : les nouveaux espaces du discours de mode », Sociétés 2009/2 (n 104), p. 105-114.

${ }^{18}$ M. Merleau-Ponty, Eloge de la philosophie et autres essais, Paris, Gallimard, coll. « Folio Essais », 1989.

${ }^{10}$ D. Osório Severo, Helena Shimizu, «Mouvements sociaux et Facebook : notes sur la technosophie », Sociétés 2016/1 (n 131), p. 107-118. DOI 10.3917/soc.131.0107
} 
partie?

Pour conclure, force est de constater que la mode digitale se situe dans une structure d'ensemble, espace au sein duquel Paolo Amaldi indique qu'il existe un tiraillement constant entre expériences subjectives et formalisations de sujets réinventant la perspective de leurs actions par le jeu des regards. ${ }^{20}$ Espace et frontière, visible et invisible, ${ }^{21}$ intime et public, ne peuvent donc s'affranchir de délimitations, qu'elles soient éthiques, idéologiques ou encore symboliques,,$^{22}$ alors que le processus de digitalisation des individus se déploie sur l'ensemble des activités humaines, modifiant visions et façons d'être au monde, s'accompagnant de vulnérabilités nouvelles.

${ }^{20}$ M. Ouvrard-Servanton, L. Salesses, H. Sqalli, Repenser les frontières de l'espace numérique, Nov 2014, Fès, Maroc. Actes du colloque Frontières numériques, 2014.

${ }^{2}$ M. Merleau-Ponty, Le visible et l'invisible, Gallimard, Paris, 1964.

${ }^{2}$ B. Arruabarrena, Identité augmentée. Les pratiques numériques de quantification de soi comme milieu de médiation identitaire. In I. Saleh, N. Bouhaï, H. Hachour (Ed.), Les frontières numériques (pp.49-72). Paris : L'Harmattan, 2014. 
Amaldi, P., Espaces. Paris : Editions de la Villette, 2007.

Arruabarrena, B., Identité augmentée. Les pratiques numériques de quantification de soi comme milieu de médiation identitaire. In I. Saleh, N. Bouhaï, H. Hachour (Ed.), Les frontières numériques (pp.49-72), l'Harmattan, Paris, 2014.

Barthes, R. Système de la mode, Editions du Seuil, Paris, 1963.

Charaudeau, P., Les stéréotypes, c'est bien. Les imaginaires, c'est mieux. Dans H. Boyer (Ed.), Stéréotypages, stéréotypes : fonctionnements ordinaires et mises en scène, l'Harmattan, Paris, 2007.

Coutant, E., Pour une courte sociologie de la mode, dans L. Salesses (dir.), Management et Marketing de la Mode, Dunod, Paris, 2013.

Ferraris, M., Mobilisation totale, PUF, Paris, 2016.

Grassi, V., Introduction à la sociologie de l'imaginaire. Une compréhension de la vie quotidienne. Erès , Ramonville Saint-Agne, 2005.

Forse, M., Les réseaux sociaux chez Simmel : les fondements d'un modèle individualiste et structural, dans L. Deroche Gurcel et P. Watier (dir.), La Sociologie de Georges Simmel, PUF, Paris, 2002.

Maffesoli, M., Le réenchantement du monde, Editions de la Table Ronde, Paris, 2007.

Maffesoli, M., La parole du silence, Editions du Cerf, coll. « Actualité », Paris, 2016.

M. Merleau-Ponty, Le visible et l'invisible, Gallimard, coll. « Tel », Paris, 1964.

Merleau-Ponty, M., Eloge de la philosophie et autres essais, Gallimard, coll. « Folio Essais », Paris, 1989.

Osório Severo, D. et Shimizu, H., "Mouvements sociaux et Facebook : notes sur la technosophie », Sociétés 2016/1 (n 131), p. 107-118.

Ouvrard-Servanton, M., Salesses, L., Sqalli, H., Repenser les frontières de l'espace numérique, Nov. 2014, Fès, Maroc. Actes du colloque Frontières numériques, 2014.

Proulx, S. et Kwok Choon, M.J., «L'usage des réseaux socionumériques : une intériorisation douce et progressive du contrôle social », Hermès, n 59, 2011, p. 105-111.

Rémy, E., «De «Que choisir»? à «Casseurs de pub»: Entre récupération marketing et nouvelles figures consuméristes », Décisions Marketing, 2007, n 46, pp. 37-50.

Rocamora, A. et Bartlett, D., « Blogs de mode : les nouveaux espaces du discours de mode », Sociétés 2009/2 ( $\left.{ }^{\circ} 104\right)$, p. 105-114.

Salesses, L. et Romain, D., «The imaginary and social representations generated by fashion images in women's magazines », Papers on Social Representations, 2014, Volume 23, pp. $23.1-23.2$

Salesses, L. et al., Marques de mode, influenceurs et e-réputation : vers une modélisation des relations entre capital marque, représentations et influence. Webinaire «E-réputation et influenceurs: de nouveaux enjeux pour les organisations? », colloque scientifique organisé par 
l'Observatoire des médias sociaux en relations publiques de l'université Laval, co-organisé avec l'IDETCOM de l'Université de Toulouse 1 Capitole, MICA de l'Université de Bordeaux Montaigne et Elico de SciencesPo-Lyon. Québec, Canada, 5 avril 2016.

Thomas, J., Introduction aux méthodologies de l'imaginaire, Ellipses, Paris, 1998.

Wunenburger, J.-J., L’imaginaire. PUF, Paris, 2003. 